IFT-UAM/CSIC-16-048

YITP-SB-16-23

\title{
Non-standard Neutrino Interactions in the Earth and the Flavor of Astrophysical Neutrinos
}

\author{
M. C. Gonzalez-Garcia ${ }^{\mathrm{a}, \mathrm{b}}$, Michele Maltonic ${ }^{\mathrm{c}}$, Ivan Martinez-Soler ${ }^{\mathrm{c}}$, Ningqiang Song ${ }^{\mathrm{a}}$ \\ ${ }^{a}$ C.N. Yang Institute for Theoretical Physics, SUNY at Stony Brook, Stony Brook, NY 11794-3840, USA \\ ${ }^{b}$ Institució Catalana de Recerca $i$ Estudis Avançats (ICREA), Departament d'Estructura $i$ Constituents de la Matèria and \\ ICC-UB, Universitat de Barcelona, 647 Diagonal, E-08028 Barcelona, Spain \\ ${ }^{c}$ Instituto de Física Teórica UAM/CSIC, Calle de Nicolás Cabrera 13-15, Universidad Autónoma de Madrid, Cantoblanco, \\ E-28049 Madrid, Spain
}

\begin{abstract}
We study the modification of the detected flavor content of ultra high-energy astrophysical neutrinos in the presence of non-standard interactions of neutrinos with the Earth matter. Unlike the case of new physics affecting the propagation from the source to the Earth, non-standard Earth matter effects induce a dependence of the flavor content on the arrival direction of the neutrino. We find that, within the current limits on non-standard neutrino interaction parameters, large deviations from the standard $3 \nu$ oscillation predictions can be expected, in particular for fluxes dominated by one flavor at the source. Conversely they do not give sizable corrections to the expectation of equalized flavors in the Earth for sources dominated by production via pion-muon decay-chain.
\end{abstract}

Keywords: Astrophysical neutrinos, non-standard neutrino interactions.

\section{Introduction}

The detection of ultra-high energy neutrinos of astrophysical origin in IceCube [1-4 marks the begin of high energy neutrino astronomy. From the point of view of astronomy, the main open question resides in finding the sources of such neutrinos, an issue to which many suggestions have been contributed (for a recent review see Ref. [5]). More on the astrophysical front, one also questions what type of mechanisms are at work in those sources to produce such high energy neutrino flux. To address this question the measurement of the flavor composition of the observed neutrinos acquires a special relevance. For example, for the pion-muon decay chain, which is the most frequently considered, one expects $\phi_{\mu}^{s}=2 \phi_{e}^{s}$ while $\phi_{\tau}^{s}=0$ [6] (denoting by $\phi_{\alpha}^{s}$ the neutrino flux of flavor $\nu_{\alpha}$ at source). Alternatively, if some of the muons lose energy very rapidly one would predict a single $\mu$-flavor flux while $\phi_{e}^{s}=\phi_{\tau}^{s}=0$ [7-11]. If neutrino production is dominated by neutron decay one expects also a single flavor flux but of electron neutrinos [8] so in this case $\phi_{\mu}^{s}=\phi_{\tau}^{s}=0$. Decay of charm mesons contribute a flux with equal amounts of electron and muon neutrinos, $\phi_{e}^{s}=\phi_{\mu}^{s}$ and $\phi_{\tau}^{s}=0$. If several of the above processes in the source compete, arbitrary flavor compositions of $\phi_{e}^{s}$ and $\phi_{\mu}^{s}$ are possible but still with $\phi_{\tau}=0$ [10]. If, in addition, $\nu_{\tau}$ are also produced in the source [12 14], then generically $\phi_{\alpha}^{s} \neq 0$ for $\alpha=e, \mu, \tau$.

Neutrino oscillations modify the flavor composition of the neutrino flux by the time they reach the Earth. In the context of the well established framework of $3 \nu$ oscillations these modifications are well understood

Email addresses: maria.gonzalez-garcia@stonybrook.edu (M. C. Gonzalez-Garcia), michele.maltoni@csic.es (Michele Maltoni), ivanj.m@csic.es (Ivan Martinez-Soler), ningqiang.song@stonybrook.edu (Ningqiang Song) 
and quantifiable given the present determination of the neutrino oscillation parameters. Because of this several studies to quantify the flavor composition of the IceCube events, even with the limited statistics data available, have been presented $15-22$ but the results are still inconclusive.

It is well-known that new physics (NP) effects beyond $3 \nu$ oscillations in the neutrino propagation can alter the predicted flavor composition of the flux reaching the Earth, thus making the task of elucidating the production mechanism even more challenging. Examples of NP considered in the literature include Lorentz or CPT violation [23, neutrino decay [24, 25, quantum decoherence [26, 27] pseudo-Dirac neutrinos [28, 29], sterile neutrinos [30], non-standard neutrino interactions with dark matter [31], or generic forms of NP in the propagation from the source to the Earth parametrized by effective operators 32 . Besides modifications of the flavor ratios many of these NP effects also induce a modification of the energy spectrum of the arriving neutrinos.

In this paper we consider an alternative form of NP, namely the possibility of non-standard interactions (NSI) of the neutrinos in the Earth matter. Unlike the kind of NP listed above, this implies that neutrinos reach the Earth surface in the expected flavor combinations provided by the "standard" $3 \nu$ vacuum oscillation mechanism: in other words, NSI in the Earth affect only the flavor evolution of the neutrino ensemble from the entry point in the Earth matter to the detector. The goal of this paper is to quantify the modification of the neutrino flavor composition at the detector because of this effect within the presently allowed values of the NSI parameters. To this aim we briefly review in Sec. 2 the formalism employed and derive the relevant flavor transition probabilities from the source to the detector including the effect of NSI in the Earth. We show that the resulting probabilities are energy independent while they depend on the zenith angle arrival direction of the neutrinos, in contrast with NP affecting propagation from the source to the Earth. Our quantitative results are presented in Sec. 3, where in particular we highlight for which source flavor composition the Earth-matter NSI can be most relevant. Finally in Sec. 4 we draw our conclusions.

\section{Formalism}

Our starting point is the initial neutrino (antineutrino) fluxes at the production point in the source which we denote as $\phi_{\alpha}^{s}\left(\bar{\phi}_{\alpha}^{s}\right)$ for $\alpha=e, \nu, \tau$. The corresponding fluxes of a given flavor at the Earth's surface are denoted as $\phi_{\alpha}^{\oplus}\left(\bar{\phi}_{\alpha}^{\oplus}\right)$ while the fluxes arriving at the detector after traversing the Earth are $\phi_{\alpha}^{d}\left(\bar{\phi}_{\alpha}^{d}\right)$. They are generically given by

$$
\phi_{\beta}^{\oplus}(E)=\sum_{\alpha} \int d E^{\prime} \mathcal{P}_{\alpha \beta}^{s \rightarrow \oplus}\left(E, E^{\prime}\right) \phi_{\alpha}^{s}\left(E^{\prime}\right), \quad \phi_{\beta}^{d}(E)=\sum_{\alpha} \int d E^{\prime} \mathcal{P}_{\alpha \beta}^{s \rightarrow d}\left(E, E^{\prime}\right) \phi_{\alpha}^{s}\left(E^{\prime}\right)
$$

and correspondingly for antineutrinos. $\mathcal{P}$ is the flavor transition probability including both coherent and incoherent effects in the neutrino propagation.

\subsection{Coherent effects}

Let us start by considering first only the coherent evolution of the neutrino ensemble. In this case, the flavor transition probabilities from the source $(s)$ to the Earth entry point $(\oplus)$ and to the detector $(d)$ can be written as

$$
\begin{array}{lll}
\mathcal{P}_{\alpha \beta}^{s \rightarrow \oplus}\left(E, E^{\prime}\right)=P_{\alpha \beta}^{s \rightarrow \oplus}(E) \delta\left(E-E^{\prime}\right), & \text { with } & P_{\alpha \beta}^{s \rightarrow \oplus}(E)=\left|A_{\alpha \beta}^{s \rightarrow \oplus}(E)\right|^{2} \\
\mathcal{P}_{\alpha \beta}^{s \rightarrow d}\left(E, E^{\prime}\right)=P_{\alpha \beta}^{s \rightarrow d}(E) \delta\left(E-E^{\prime}\right), & \text { with } & P_{\alpha \beta}^{s \rightarrow d}(E)=\left|A_{\alpha \beta}^{s \rightarrow d}(E)\right|^{2}=\left|\sum_{\gamma} A_{\alpha \gamma}^{s \rightarrow \oplus} A_{\gamma \beta}^{\oplus \rightarrow d}\right|^{2},
\end{array}
$$

where we have introduced the flavor transition amplitude from the source to the Earth surface $A^{s \rightarrow \oplus}$ and from the Earth surface to the detector $A^{\oplus \rightarrow d}$.

Generically these amplitudes are obtained by solving the neutrino and antineutrino evolution equations for the flavor wave function $\vec{\nu}(x)=\left\{\nu_{e}(x), \nu_{\mu}(x), \nu_{\tau}(x)\right\}^{T}$

$$
i \frac{d \vec{\nu}(x)}{d x}=H_{\nu}^{s \rightarrow \oplus} \vec{\nu}(x), \quad i \frac{d \vec{\nu}(x)}{d x}=H_{\bar{\nu}}^{s \rightarrow \oplus} \vec{\nu}(x)
$$


for evolution between the source and the Earth surface and

$$
i \frac{d \vec{\nu}(x)}{d x}=H_{\nu}^{\oplus \rightarrow d} \vec{\nu}(x), \quad i \frac{d \vec{\nu}(x)}{d x}=H_{\bar{\nu}}^{\oplus \rightarrow d} \vec{\nu}(x),
$$

for evolution in the Earth matter.

In this work we are interested in standard vacuum oscillation dominating the propagation from the source to the detector but allowing for new physics in the interactions of the neutrinos in the Earth matter. In this case

$$
H_{\nu}^{s \rightarrow \oplus}=\left(H_{\bar{\nu}}^{s \rightarrow \oplus}\right)^{*}=H_{\mathrm{osc}}=U D_{\mathrm{vac}} U^{\dagger} \quad \text { with } \quad D_{\mathrm{vac}}=\frac{1}{2 E} \operatorname{diag}\left(0, \Delta m_{21}^{2}, \Delta m_{31}^{2}\right)
$$

and $U$ is the leptonic mixing matrix [33, 34. While

$$
H_{\nu}^{\oplus \rightarrow d} \simeq H_{\mathrm{mat}}, \quad H_{\bar{\nu}}^{\oplus \rightarrow d} \simeq-H_{\mathrm{mat}}^{*}
$$

where the $\simeq$ corresponds to neglecting vacuum oscillations inside the Earth which is a very good approximation for the relevant neutrino energies $(\gtrsim 1 \mathrm{TeV})$.

The standard theoretical framework for the NP considered here is provided by non-standard interactions affecting neutrino interactions in the Earth matter. They can be described by effective four-fermion operators of the form

$$
\mathcal{L}_{\mathrm{NSI}}=-2 \sqrt{2} G_{F} \varepsilon_{\alpha \beta}^{f P}\left(\bar{\nu}_{\alpha} \gamma^{\mu} \nu_{\beta}\right)\left(\bar{f} \gamma_{\mu} P f\right),
$$

where $f$ is a charged fermion, $P=(L, R)$ and $\varepsilon_{\alpha \beta}^{f P}$ are dimensionless parameters encoding the deviation from standard interactions. NSI enter in neutrino propagation only through the vector couplings, so in the most general case the non-standard matter Hamiltonian can be parametrized as [35]

$$
H_{\mathrm{mat}}=\sqrt{2} G_{F} N_{e}(r)\left(\begin{array}{ccc}
1 & 0 & 0 \\
0 & 0 & 0 \\
0 & 0 & 0
\end{array}\right)+\sqrt{2} G_{F} \sum_{f=e, u, d} N_{f}(r)\left(\begin{array}{ccc}
\varepsilon_{e e}^{f} & \varepsilon_{e \mu}^{f} & \varepsilon_{e \tau}^{f} \\
\varepsilon_{e \mu}^{f *} & \varepsilon_{\mu \mu}^{f} & \varepsilon_{\mu \tau}^{f} \\
\varepsilon_{e \tau}^{f *} & \varepsilon_{\mu \tau}^{f *} & \varepsilon_{\tau \tau}^{f}
\end{array}\right) .
$$

The standard model interactions are encoded in the non-vanishing ee entry in the first term of Eq. (9), while the non-standard interactions with fermion $f$ are accounted by the $\varepsilon_{\alpha \beta}^{f}$ coefficients with $\varepsilon_{\alpha \beta}^{f}=\varepsilon_{\alpha \beta}^{f L}+\varepsilon_{\alpha \beta}^{f R}$. Here $N_{f}(r)$ is the number density of fermions $f$ in the Earth matter. In practice, the PREM model [36] fixes the neutron/electron ratio to $Y_{n}=1.012$ in the Mantle and $Y_{n}=1.137$ in the Core, with an average $Y_{n}=1.051$ all over the Earth. Thus we get an average up-quark/electron ratio $Y_{u}=3.051$ and downquark/electron ratio $Y_{d}=3.102$. We can therefore define:

$$
\varepsilon_{\alpha \beta} \equiv \sum_{f=e, u, d}\left\langle\frac{Y_{f}}{Y_{e}}\right\rangle \varepsilon_{\alpha \beta}^{f}=\varepsilon_{\alpha \beta}^{e}+Y_{u} \varepsilon_{\alpha \beta}^{u}+Y_{d} \varepsilon_{\alpha \beta}^{d}
$$

so that the matter part of the Hamiltonian can be written as:

$$
H_{\mathrm{mat}}=\sqrt{2} G_{F} N_{e}(r)\left(\begin{array}{ccc}
1+\varepsilon_{e e} & \varepsilon_{e \mu} & \varepsilon_{e \tau} \\
\varepsilon_{e \mu}^{*} & \varepsilon_{\mu \mu} & \varepsilon_{\mu \tau} \\
\varepsilon_{e \tau}^{*} & \varepsilon_{\mu \tau}^{*} & \varepsilon_{\tau \tau}
\end{array}\right) \equiv W D_{\mathrm{mat}} W^{\dagger}
$$

where

$$
D_{\text {mat }}=\sqrt{2} G_{F} N_{e}(r) \operatorname{diag}\left(\varepsilon_{1}, \varepsilon_{2}, \varepsilon_{3}\right) .
$$

where $W$ is a $3 \times 3$ unitary matrix containing six physical parameters, three real angles and three complex phases. So without loss of generality the matter potential contains eight parameters, five real and three phases (as only difference of $\varepsilon_{i}$ enter the flavor transition probabilities, only differences in the $\varepsilon_{\alpha \alpha}$ are physically relevant for neutrino oscillation data). 


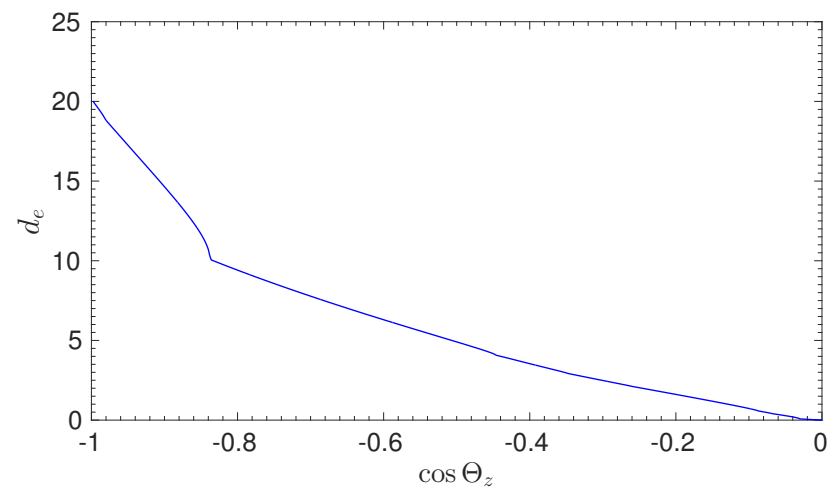

Figure 1: The normalized density integral $d_{e}$ along the neutrino path as a function of the neutrino arrival zenith angle.

Altogether the flavor transition probabilities from a source at distance $L$ are

$$
\begin{aligned}
& P_{\alpha \beta}^{s \rightarrow d}(E)=\sum_{\gamma \eta k l} W_{\beta k} W_{\beta l}^{*} W_{\gamma l} W_{\eta k}^{*} \exp \left(-i d_{e} \Delta \varepsilon_{k l}\right) \sum_{i j} U_{\eta i} U_{\gamma j}^{*} U_{\alpha j} U_{\alpha i}^{*} \exp \left(-i \frac{\Delta m_{i j}^{2}}{2 E} L\right), \\
& P_{\alpha \beta}^{s \rightarrow \oplus}(E)=\sum_{i j} U_{\beta i} U_{\beta j}^{*} U_{\alpha j} U_{\alpha i}^{*} \exp \left(-i \frac{\Delta m_{i j}^{2}}{2 E} L\right)
\end{aligned}
$$

where $\Delta \varepsilon_{k l}=\varepsilon_{k}-\varepsilon_{l}$. Since for astrophysical neutrinos the propagation distance $L$ is much longer than the oscillation wavelength, we can average out the vacuum oscillation terms:

$$
\begin{aligned}
P_{\alpha \beta}^{s \rightarrow d}(E)= & \sum_{i}\left|U_{\alpha i}\right|^{2}\left|U_{\beta i}\right|^{2}-2 \sum_{\gamma \eta k l i} \operatorname{Re}\left(W_{\beta k} W_{\beta l}^{*} W_{\gamma l} W_{\eta k}^{*} U_{\eta i} U_{\gamma i}^{*}\left|U_{\alpha i}\right|^{2}\right) \sin ^{2}\left(d_{e} \frac{\Delta \varepsilon_{k l}}{2}\right) \\
& +\sum_{\gamma \eta k l i} \operatorname{Im}\left(W_{\beta k} W_{\beta l}^{*} W_{\gamma l} W_{\eta k}^{*} U_{\eta i} U_{\gamma i}^{*}\left|U_{\alpha i}\right|^{2}\right) \sin \left(d_{e} \Delta \varepsilon_{k l}\right), \\
P_{\alpha \beta}^{s \rightarrow \oplus}(E)= & \sum_{i}\left|U_{\alpha i}\right|^{2}\left|U_{\beta i}\right|^{2} .
\end{aligned}
$$

In these expressions we have introduced the dimensionless normalization for the matter potential integral along the neutrino trajectory in the Earth

$$
d_{e}\left(\Theta_{z}\right) \equiv \int_{0}^{2 R \cos \left(\pi-\Theta_{z}\right)} \sqrt{2} G_{F} N_{e}(r) d x, \quad \text { with } \quad r=\sqrt{R_{\oplus}^{2}+x^{2}+2 R_{\oplus} x \cos \Theta_{z}},
$$

which we plot in Fig. 1. The integral includes both the effect of the increase length of the path in the Earth and the increase average density which is particular relevant for trajectories crossing the core and leads to the higher slope of the curve for $\cos \Theta_{z} \lesssim-0.84$.

We notice that the total coherent flavor transition probability remains energy independent even in the presence of NSI. Also the last term in Eq. 15 does not change sign for antineutrinos since both the imaginary part of the combination of mixing matrices and the phase of the oscillating sin change sign for antineutrinos 1 In other words, there is no CP violation even if all the phases in $U$ and $W$ are kept different from zero. These two facts render the flavor composition of the fluxes at the detector independent of the energy spectrum and the neutrino/antineutrino ratio at the source, as long as the flavor composition at the source is the same for both neutrinos and antineutrinos. This is just as the case for standard $3 \nu$ oscillations in the absence of $\mathrm{NP}^{2}$

\footnotetext{
${ }^{1}$ Indeed this term preserves CP but violates time reversal, as it is well known that Earth matter effects violate CPT.

${ }^{2}$ Relaxing the assumption of equal flavor composition for neutrinos and antineutrinos at the source can lead to additional interesting effects even in the case of standard oscillations as discussed in Ref. [37 38.
} 
In brief, the effect of NSI in the Earth is to modify the flavor composition at the detector as compared to the standard case, in a way which depends on the zenith angle of the arrival direction of the neutrinos. Also, as expected, the effect only appears in presence of additional flavor mixing during propagation in the Earth, i.e., for $W_{\alpha i} \neq C \delta_{\alpha i}$, which occurs only if some off-diagonal $\varepsilon_{\alpha \beta}$ (with $\alpha \neq \beta$ ) is different from zero.

\subsection{Incoherent effects}

In addition to the coherent effects discussed so far, high-energy neutrinos propagating through the Earth can also interact inelastically with the Earth matter either by charged current or by neutral current interactions. As a consequence of these inelastic processes the neutrino flux is attenuated, its energy is degraded, and secondary fluxes are generated from the decay of the charged leptons (in particular $\tau^{ \pm}$) produced in charged current interactions. In some new physics scenario attenuation and other decoherence effects can also occur in the travel from the source to the Earth, but they are not relevant for this work.

For simplicity, let us first neglect NSI and focus only on the usual $3 \nu$ oscillation framework. In the standard scenario, attenuation and regeneration effects can be consistently described by a set of coupled partial integro-differential cascade equations (see for example [39] and references therein). In this case the fluxes at the arrival point in the Earth are given by Eq. (1) and 22 while for the fluxes at the detector we have:

$$
\mathrm{SM}: \quad \mathcal{P}_{\alpha \beta}^{s \rightarrow d}\left(E, E^{\prime}\right)=\sum_{\gamma} P_{\alpha \gamma}^{s \rightarrow \oplus}(E) F_{\gamma \beta}^{\oplus \rightarrow d}\left(E, E^{\prime}\right)
$$

where $F_{\gamma \beta}^{\oplus \rightarrow d}\left(E, E^{\prime}\right)$ is the function accounting for attenuation and regeneration effects, which depends on the trajectory of the neutrino in the Earth matter (i.e., it depends on $\Theta_{z}$ ). Attenuation is the dominant effect and for most energies is only mildly flavor dependent. So the dominant incoherent effects verify

$$
\mathrm{SM}: \quad F_{\gamma \beta}^{\oplus \rightarrow d}\left(E, E^{\prime}\right) \simeq \delta_{\gamma \beta} F_{\mathrm{att}}^{\oplus \rightarrow d}(E) \delta\left(E-E^{\prime}\right) .
$$

When considering NSI in the Earth the simple factorization of coherent and incoherent effects introduced in Eq. (18) does not hold, since NSI-induced oscillations, attenuation, and regeneration occur simultaneously while the neutrino beam is traveling across the Earth's matter. In order to properly account for all these effects we need to replace the evolution equation in the Earth (5) with a more general expression including also the incoherent components. This can be done by means of the density matrix formalism, as illustrated in Ref. [40] (see also Ref. [41]). However, if one neglects the subleading flavor dependence of these effects and focus only on the dominant attenuation term, as we did in Eq. (19) for the standard case, it becomes possible to write even in the presence of NSI-oscillations:

$$
\mathrm{NSI}: \quad \mathcal{P}_{\alpha \beta}^{s \rightarrow d}\left(E, E^{\prime}\right) \simeq P_{\alpha \beta}^{s \rightarrow d}(E) F_{\mathrm{att}}^{\oplus \rightarrow d}(E) \delta\left(E-E^{\prime}\right)
$$

with $P_{\alpha \beta}^{s \rightarrow d}(E)$ given in Eq. 15. In other words, although the presence of NSI affects the flavor composition at the detector through a modification of the coherent part of the evolution in the Earth, the incoherent part is practically the same in both the standard and the non-standard case and does not introduce relevant flavor distortions.

In the next section we quantify our results taking into account the existing bounds on NSI. For simplicity we will consider only NSI with quarks and we further assume that the NSI Hamiltonian is real. At present the strongest model-independent constraints on NSI with quarks relevant to neutrino propagation arise from the global analysis of oscillation data [35, 42. (see also [43]) in combination with some constraints from scattering experiments [44, 45] such as CHARM [46, 47], CDHSW [48] and NuTeV [49]. As shown in Ref. 35. neutrino oscillations provide the stronger constraints on NSI, with the exception of some large $\varepsilon_{e e}-\varepsilon_{\mu \mu}$ terms which are still allowed in association with a flip of the octant of $\theta_{12}$, the so-called "dark-side" solution (or LMA-D) found in Ref. [43. However, these large NSI's are disfavored by scattering data [43. A fully consistent analysis of both oscillation and scattering data covering the LMA-D region is still missing, so here we conservatively consider only NSI's which are consistent with oscillations within the LMA regions. 
The corresponding allowed ranges read (we quote the most constraining of both $u$ and $d$ NSI's):

\begin{tabular}{ccc} 
& $90 \% \mathrm{CL}$ & $3 \sigma \mathrm{CL}$ \\
\hline$\varepsilon_{e e}^{q}-\varepsilon_{\mu \mu}^{q}$ & {$[+0.02,+0.51]$} & {$[-0.09,+0.71]$} \\
$\varepsilon_{\tau \tau}^{q}-\varepsilon_{\mu \mu}^{q}$ & {$[-0.01,+0.03]$} & {$[-0.03,+0.19]$} \\
$\varepsilon_{e \mu}^{q}$ & {$[-0.09,+0.04]$} & {$[-0.16,+0.11]$} \\
$\varepsilon_{e \tau}^{q}$ & {$[-0.13,+0.14]$} & {$[-0.38,+0.29]$} \\
$\varepsilon_{\mu \tau}^{q}$ & {$[-0.01,+0.01]$} & {$[-0.03,+0.03]$}
\end{tabular}

where for each NSI coupling the ranges are shown after marginalization over all the oscillations parameters and the other NSI couplings.

\section{Results}

Flavor composition of the astrophysical neutrinos are usually parametrized in terms of the flavor ratios at the source and at the Earth surface, defined as:

$$
\xi_{\alpha}^{s} \equiv \frac{\phi_{\alpha}^{s}(E)}{\sum_{\gamma} \phi_{\gamma}^{s}(E)}, \quad \xi_{\beta}^{\oplus} \equiv \frac{\phi_{\beta}^{\oplus}(E)}{\sum_{\gamma} \phi_{\gamma}^{\oplus}(E)}=\sum_{\alpha} P_{\alpha \beta}^{s \rightarrow \oplus}(E) \xi_{\alpha}^{s}
$$

and it has become customary to plot them in ternary plots. Experimentally $\xi_{\beta}^{\oplus}$ are reconstructed from the measured neutrino fluxes in the detector $\phi_{\alpha}^{d}$ by deconvoluting the incoherent effects due to SM interactions in the Earth matter:

$$
\xi_{\beta}^{\oplus, \text { rec }} \equiv \frac{\phi_{\beta}^{\oplus, \text { rec }}(E)}{\sum_{\gamma} \phi_{\gamma}^{\oplus, \text { rec }}(E)} \quad \text { with } \quad \phi_{\beta}^{\oplus, \text { rec }}(E) \equiv \sum_{\gamma} \int d E^{\prime} G_{\gamma \beta}^{\oplus \leftarrow d}\left(E, E^{\prime}\right) \phi_{\gamma}^{d}\left(E^{\prime}\right)
$$

where the function $G_{\alpha \beta}^{\oplus \leftarrow d}\left(E, E^{\prime}\right)$ is the inverse of the Earth attenuation + degradation + regeneration function $F_{\alpha \beta}^{\oplus \rightarrow d}\left(E, E^{\prime}\right)$ introduced in the previous section:

$$
\sum_{\gamma} \int d E^{\prime \prime} F_{\gamma \beta}^{\oplus \rightarrow d}\left(E, E^{\prime \prime}\right) G_{\alpha \gamma}^{\oplus \leftarrow d}\left(E^{\prime \prime}, E^{\prime}\right)=\delta_{\alpha \beta} \delta\left(E-E^{\prime}\right)
$$

Under the approximation described in Eq. 19] $G_{\alpha \beta}^{\oplus \leftarrow d}\left(E, E^{\prime}\right)$ reduces to:

$$
G_{\alpha \beta}^{\oplus \leftarrow d}\left(E, E^{\prime}\right) \simeq \delta_{\alpha \beta} \frac{1}{F_{\mathrm{att}}^{\oplus \rightarrow d}(E)} \delta\left(E-E^{\prime}\right)
$$

so that

$$
\xi_{\beta}^{\oplus, \text { rec }} \simeq \frac{\phi_{\beta}^{d}(E) / F_{\mathrm{att}}^{\oplus \rightarrow d}(E)}{\sum_{\gamma} \phi_{\gamma}^{d}(E) / F_{\mathrm{att}}^{\oplus \rightarrow d}(E)}=\frac{\phi_{\beta}^{d}(E)}{\sum_{\gamma} \phi_{\gamma}^{d}(E)} \equiv \xi_{\beta}^{d}
$$

where we have introduced the flavor ratios at the detector $\xi_{\beta}^{d}$. Thus we have shown that the reconstructed flavor ratios at the surface of the Earth $\left(\xi_{\beta}^{\oplus, \text { rec }}\right)$ are well approximated by the measured flavor ratios at the detector $\left(\xi_{\beta}^{d}\right)$. This conclusion depends only on the validity of the approximation $(19)$, and therefore applies both for standard oscillations and in the presence of new physics such as Earth NSI. It should be noted, however, that in the standard case $\xi_{\beta}^{\oplus, \text { rec }}$ really coincides with the actual flavor ratios $\xi_{\beta}^{\oplus}$ defined in Eq. 22, whereas in the presence of NSI this is no longer the case.

In what follows we will present our results in terms of flavor ratios at the detector $\xi_{\beta}^{d}$, since, as we have just seen, they are good estimators of the reconstructed quantities $\xi_{\beta}^{\oplus \text {,rec }}$ usually shown by the experimental collaborations. It is easy to show that:

$$
\xi_{\beta}^{d}=\sum_{\alpha} P_{\alpha \beta}^{s \rightarrow d}(E) \xi_{\alpha}^{s}
$$



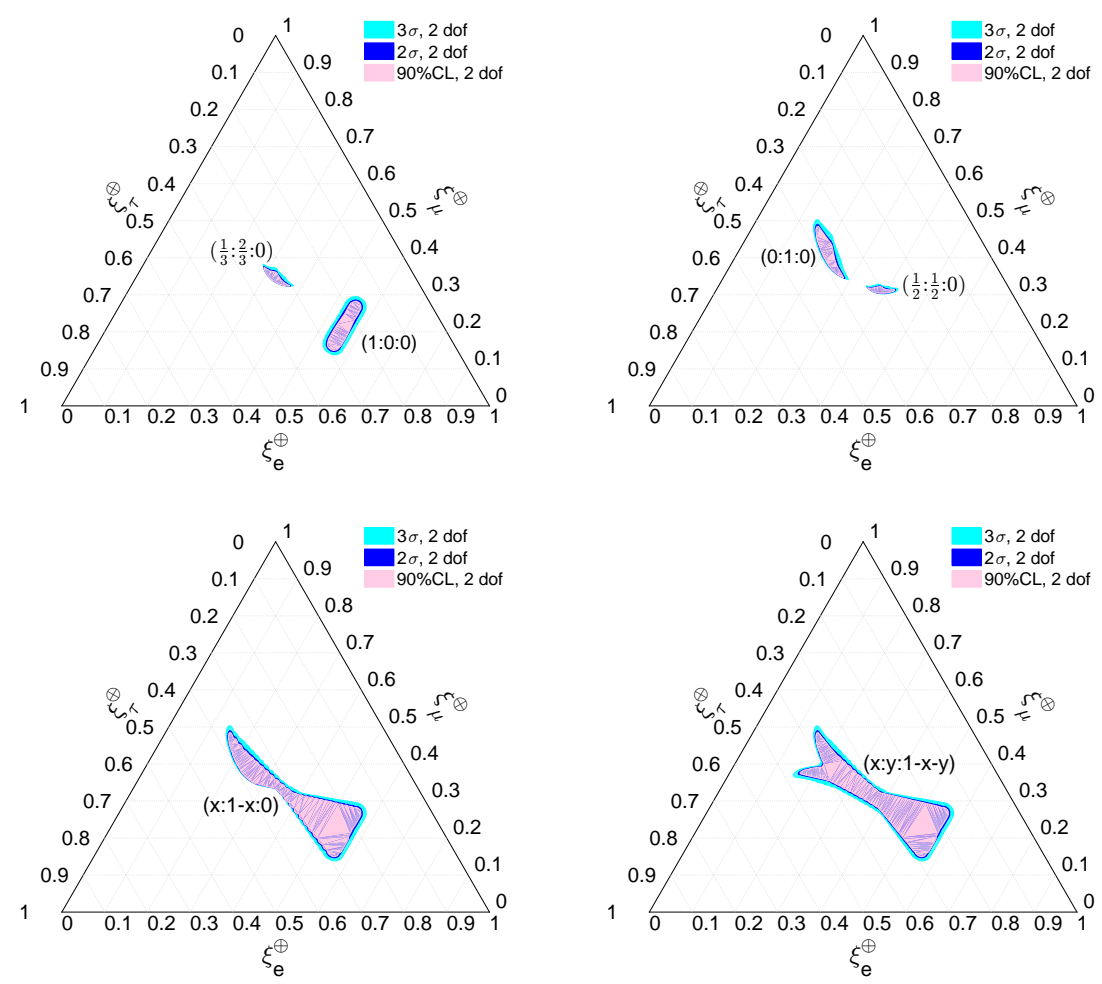

Figure 2: Two-dimensional projections of the allowed regions from the global analysis of oscillation data from Ref. 50] in the relevant combinations giving the flavor content at the Earth. The allowed regions are shown at $90 \%, 95 \%$ and $3 \sigma$ CL. In the upper panels we show the regions for four initial flavor compositions $\left(\xi_{e}^{s}: \xi_{\mu}^{s}: \xi_{\tau}^{s}\right)=\left(\frac{1}{3}: \frac{2}{3}: 0\right),(1: 0: 0),(0: 1: 0)$, and $\left(\frac{1}{2}: \frac{1}{2}: 0\right)$. In the lower panel the regions are shown for the more general scenarios, $\left(\xi_{e}^{s}: \xi_{\mu}^{s}: \xi_{\tau}^{s}\right)=(x: 1-x: 0)$ for $0 \leq x \leq 1$, and $\left(\xi_{e}^{s}: \xi_{\mu}^{s}: \xi_{\tau}^{s}\right)=(x: y: 1-x-y)$ for $0 \leq x, y \leq 1$.

where $P_{\alpha \beta}^{s \rightarrow d}(E)$ is obtained from Eq. 15. In principle, one may expect that the flavor ratios $\xi_{\beta}^{d}$ would depend on the neutrino energy, either through the oscillation probability $P_{\alpha \beta}^{s \rightarrow d}(E)$ or though the intrinsic energy dependence of the flavor ratios at the source $\xi_{\alpha}^{s}$. However, as we have seen in the previous section the expression in Eq. (15) is independent of $E$, and moreover we will assume (as it is customary to do) that the ratios $\xi_{\alpha}^{s}$ do not depend on the neutrino energy even though the fluxes $\phi_{\alpha}^{s}(E)$ do. Hence, the flavor ratios $\xi_{\beta}^{d}$ are independent of energy and they can be conveniently plotted in a ternary plot.

Let us now discuss the results of our fit, starting with the simpler case of standard oscillations. In the absence of new physics effects the present determination of the leptonic mixing matrix from the measurements of neutrino oscillation experiments allows us to determine the astrophysical neutrino flavor content at detection given an assumption of the neutrino production mechanism. For completeness and reference we show in Fig. 2 the allowed regions of the flavor ratios at the Earth as obtained from the projection of the six oscillation parameter $\chi^{2}$ function of the global NuFIT analysis of oscillation data [50, 51] in the relevant mixing combinations (see also [16, 32, 38). We stress that in our plots the correlations among the allowed ranges of the oscillation parameters in the full six-parameter space are properly taken into account. The results are shown after marginalization over the neutrino mass ordering and for different assumptions of the flavor content at the source as labeled in the figure. Fig. 2 illustrates the well-known fact [6] that during propagation from the source neutrino oscillations lead to flavor content at the Earth close to $\left(\xi_{e}^{\oplus}: \xi_{\mu}^{\oplus}: \xi_{\tau}^{\oplus}\right)=\left(\frac{1}{3}: \frac{1}{3}: \frac{1}{3}\right)$, with largest deviations for the case when the flavor content at the source is $(1: 0: 0) 52$ and $(0: 1: 0)$.

As discussed in the previous section NSI in the Earth modify these predictions and, unlike for NP effects in the propagation from the source, such Earth-induced modifications are a function of the arrival zenith 


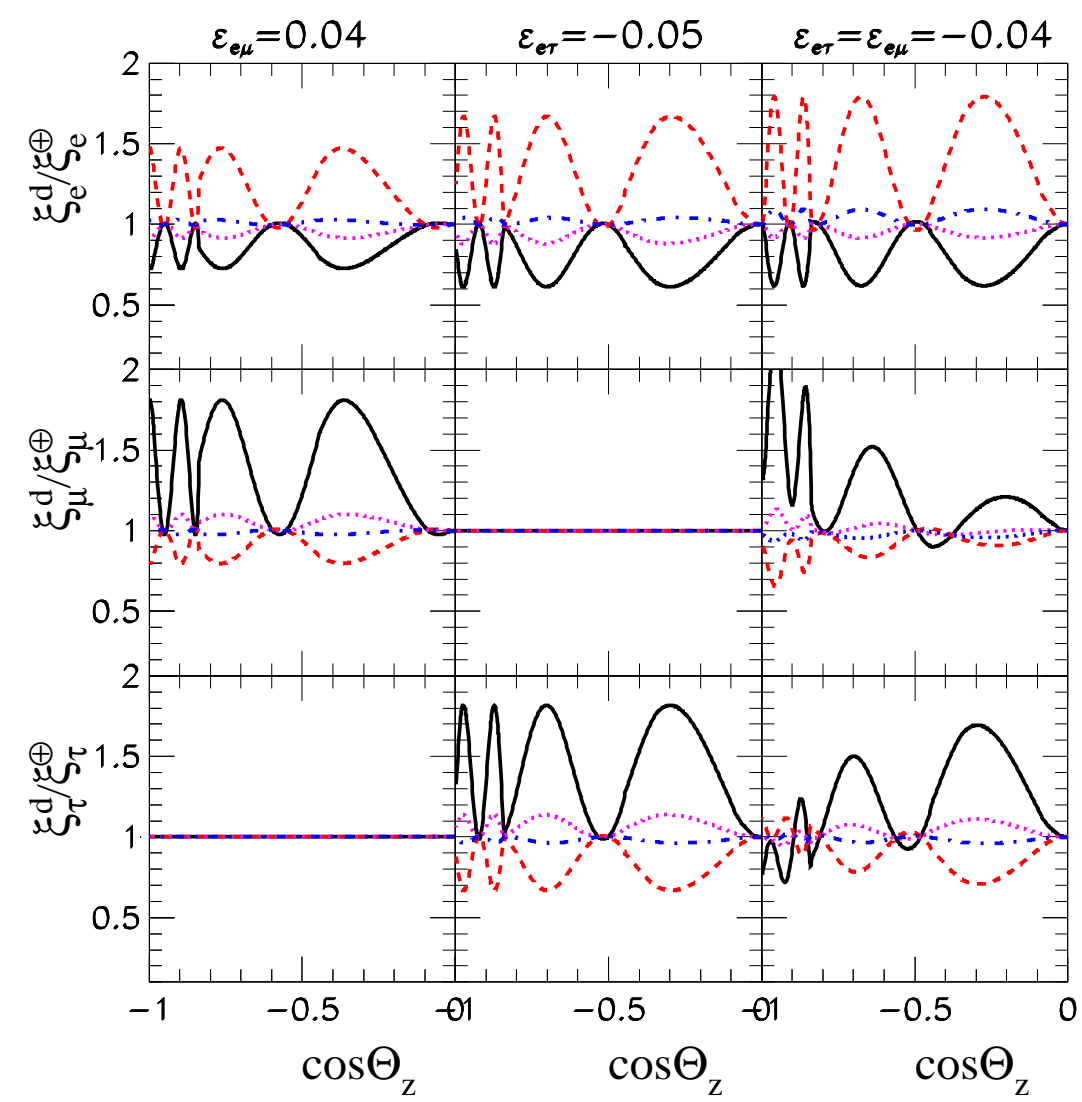

Figure 3: Flavor ratios at the detector as a function of the zenith angle of the neutrino normalized to the expectation in the absence of NSI and for oscillation parameters at the best fit of the global analysis $\left(\sin ^{2} \theta_{12}=0.305, \sin ^{2} \theta_{13}=0.0219\right.$, $\sin ^{2} \theta_{23}=0.579$, and $\left.\delta_{\mathrm{CP}}=254^{\circ}\right)$. For the left (central) [right] panels the only non-vanishing NSI parameters are $\varepsilon_{e \mu}=0.04$ $\left(\varepsilon_{e \tau}=-0.05\right)\left[\varepsilon_{e \mu}=\varepsilon_{e \tau}=-0.04\right]$. The different curves corresponds to different flavor composition at the source: ( $\xi_{e}^{s}: \xi_{\mu}^{s}$ : $\left.\xi_{\tau}^{s}\right)=(1: 0: 0)$ (full black), $(0: 1: 0)$ (dashed red), $\left(\frac{1}{2}: \frac{1}{2}: 0\right)$ (dotted blue), and $\left(\frac{1}{3}: \frac{2}{3}: 0\right)$ (dash-dotted purple).

angle of the neutrino. As illustration we show in Fig. 3 the variation of the flavor ratios at the detector as a function of the zenith angle of the neutrino for some values of the $\varepsilon_{\alpha \beta}$ well within the presently allowed $90 \%$ CL ranges. In our convention $\cos \Theta_{z}=-1$ corresponds to vertically upcoming neutrinos (which have crossed the whole Earth before reaching the detector) while $\cos \Theta_{z}=0$ corresponds to horizontally arriving neutrinos (for which effectively no Earth matter is crossed so that $\left.\xi_{\beta}^{d}\left(\cos \Theta_{z}=0\right)=\xi_{\beta}^{\oplus}\right)$. From Fig. 3 we can observe the main characteristics of the effect of NSI in the Earth matter. Deviations are sizable for flavor $\alpha$ as long as $\varepsilon_{\beta \neq \alpha}$ is non-zero and $\xi_{\alpha}^{s}$ or $\xi_{\beta}^{s}$ are non-zero. Larger effects are expected for source flavor compositions for which vacuum oscillations from the source to the Earth lead to "less equal" ratios at the Earth surface: $(1: 0: 0)$ and $(0: 1: 0)$. Finally the increase in frequency for almost vertical neutrino direction is a consequence of the increase of the integral density $d_{e}$ for core crossing trajectories (see Fig. 1).

Next we show how the allowed regions in the ternary plots shown in Fig. 2 are modified when including the effect of the NSI presently allowed at given CL. In order to do so we project the $\chi^{2}$ of the global analysis of oscillation data in the presence of arbitrary NSI on the relevant combinations entering in the flavor ratios within a given CL. The results are shown in Fig. 4 Fig. 5 and Fig. 6 for the flavor compositions at source $\left(\xi_{e}^{s}: \xi_{\mu}^{s}: \xi_{\tau}^{s}\right)=(1: 0: 0),(0: 1: 0)$ and $\left(\frac{1}{3}: \frac{2}{3}: 0\right)$, respectively. The results are shown averaged over four zenith angular directions.

Comparing the allowed regions in Fig. 4 and Fig. 5 with the corresponding ones for $(1: 0: 0)$ and $(0: 1: 0)$ compositions in the case of standard $3 \nu$ oscillations given in Fig. 2 we see that the flavor ratios can take now much wider range of values in any of the zenith angle ranges considered. Moreover, although 


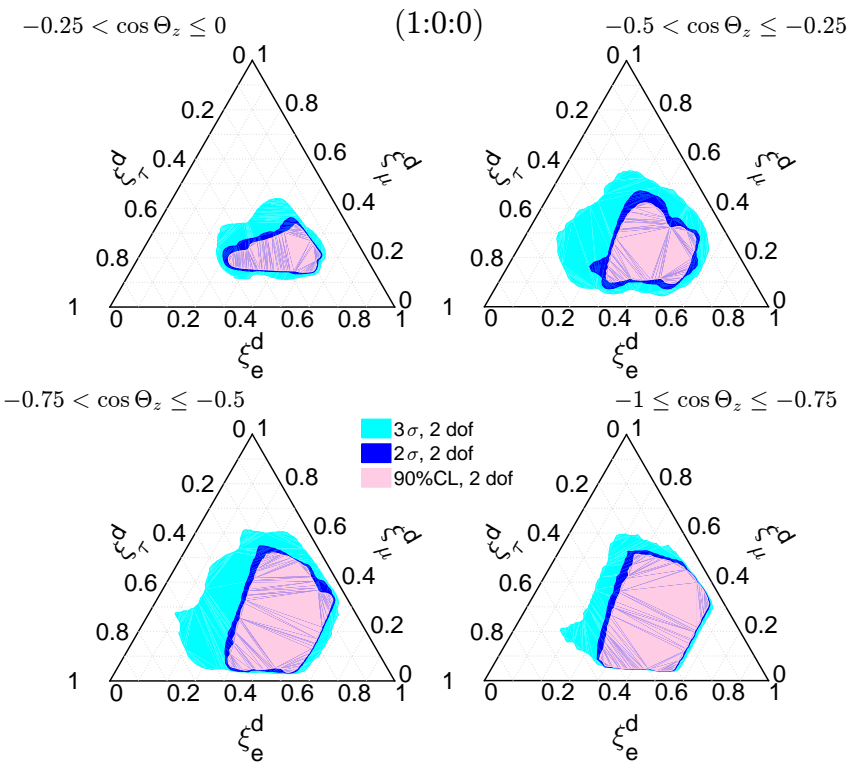

Figure 4: Allowed regions for the flavor ratios in the presence of NSI in the Earth at 90, 95\% and $3 \sigma$ CL for an initial flavor $\left(\xi_{e}^{s}: \xi_{\mu}^{s}: \xi_{\tau}^{s}\right)=(1: 0: 0)$. The four triangles correspond to averaging over neutrinos arriving with directions given in the range $0 \geq \cos \Theta_{z}>-0.25$ (upper left), $-0.25 \geq \cos \Theta_{z}>-0.5$ (upper right) $-0.5 \geq \cos \Theta_{z}>-0.75$ (lower left), and $-0.75 \geq \cos \Theta_{z} \geq-1$ (lower right).

sizable deviations from $\left(\xi_{e}^{d}: \xi_{\mu}^{d}: \xi_{\tau}^{d}\right)=\left(\frac{1}{3}: \frac{1}{3}: \frac{1}{3}\right)$ are possible, the allowed regions now extend to include $\left(\frac{1}{3}: \frac{1}{3}: \frac{1}{3}\right)$ at $\mathrm{CL}$ of $3 \sigma$ or lower. We also see that the larger CL region becomes smaller for most vertical arrival directions (see the relative size of the light blue regions in the two lower triangles on these figures). This is so because at those CL for the larger values of $\varepsilon$ allowed the NSI-induced oscillations are fast enough to be averaged out $\left\langle\sin ^{2}\left(\Delta \varepsilon_{i j} \frac{d_{e}}{2}\right)\right\rangle \sim \frac{1}{2}$ for those trajectories while the value in the second most vertical angular bin can be in average larger than $1 / 2$. For contrast, as illustrated in Fig. 6, for the case of flavor composition at the source $\left(\xi_{e}^{s}: \xi_{\mu}^{s}: \xi_{\tau}^{s}\right)=\left(\frac{1}{3}: \frac{2}{3}: 0\right)$ NSI in the Earth never induce sizable modifications of the expectation $\left(\xi_{e}^{d}: \xi_{\mu}^{d}: \xi_{\tau}^{d}\right)=\left(\frac{1}{3}: \frac{1}{3}: \frac{1}{3}\right)$.

\section{Conclusions}

The measurement of the flavor composition of the detected ultra-high energy neutrinos can be a powerful tool to learn about the mechanisms at work in their sources. Such inference, however, relies on the understanding of the particle physics processes relevant to the neutrino propagation from the source to the detector. The presence of NP effects beyond those of the well established mass-induced $3 \nu$ oscillations alter the flavor composition at the detector and can therefore affect the conclusions on the dominant production mechanism.

In this work we have focused on NP effects associated with NSI of the neutrinos in the Earth matter. The relevant flavor transition probabilities accounting from oscillations from the source to the Earth plus NSI in the Earth are energy independent but depend on the zenith angle of the arrival direction of the neutrinos, which is a characteristic feature of this form of NP. Quantitatively, we have shown that within the presently allowed range of NSI large deviations from the standard $3 \nu$ oscillation predictions for the detected flavor composition can be expected, in particular for fluxes dominated by one flavor at the source. On the contrary we find that the expectation of equalized flavors in the Earth for sources dominated by production via pion-muon decay-chain is robust even in the presence of this form of NP. 


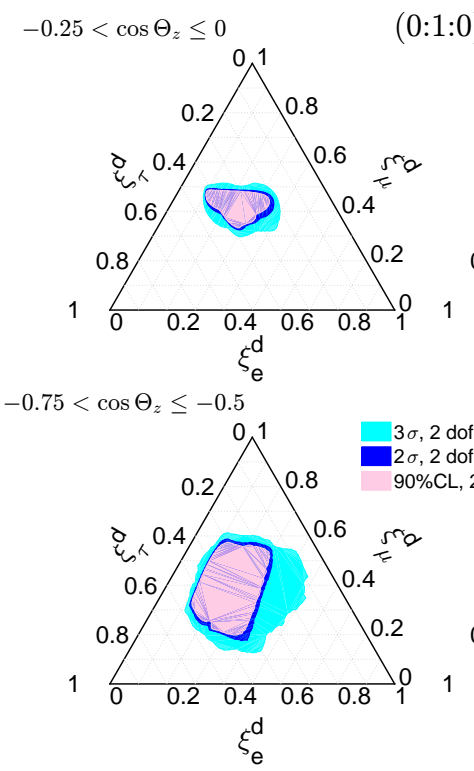

$(0: 1: 0)$
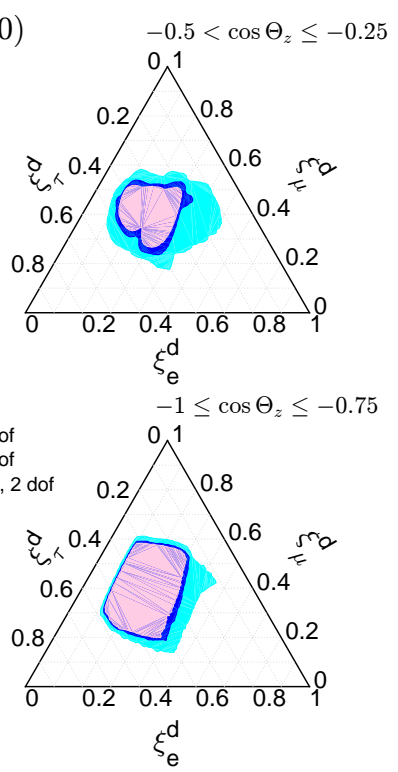

Figure 5: Same as Fig. 4 for $\left(\xi_{e}^{s}: \xi_{\mu}^{s}: \xi_{\tau}^{s}\right)=(0: 1: 0)$.

\section{Acknowledgments}

I.M.S. thanks YITP at Stony Brook Univ. for their kind hospitality during the visit that lead to this work. This work is supported by USA-NSF grant PHY-13-16617, by EU Networks FP7 ITN INVISIBLES (PITN-GA-2011-289442), FP10 ITN ELUSIVES (H2020-MSCA-ITN-2015-674896) and INVISIBLES-PLUS (H2020-MSCA-RISE-2015-690575). M.C.G-G. also acknowledges support by MINECO grants 2014-SGR104, FPA2013-46570, and "Maria de Maetzu" program grant MDM-2014-0367 of ICCUB. M.M. and I.M-S. also acknowledge support by MINECO grants FPA2012-31880, FPA2012-34694 and by the "Severo Ochoa" program grant SEV-2012-0249 of IFT.

\section{References}

[1] M. G. Aartsen, et al., First observation of PeV-energy neutrinos with IceCube, Phys. Rev. Lett. 111 (2013) 021103. arXiv:1304.5356 doi:10.1103/PhysRevLett.111.021103

[2] M. G. Aartsen, et al., Evidence for High-Energy Extraterrestrial Neutrinos at the IceCube Detector, Science 342 (2013) 1242856. arXiv: 1311.5238 doi:10.1126/science.1242856

[3] M. G. Aartsen, et al., Observation of High-Energy Astrophysical Neutrinos in Three Years of IceCube Data, Phys. Rev. Lett. 113 (2014) 101101. arXiv:1405.5303 doi:10.1103/PhysRevLett.113.101101

[4] M. G. Aartsen, et al., Evidence for Astrophysical Muon Neutrinos from the Northern Sky with IceCube, Phys. Rev. Lett. 115 (8) (2015) 081102. arXiv:1507.04005 doi:10.1103/PhysRevLett.115.081102.

[5] L. A. Anchordoqui, et al., Cosmic Neutrino Pevatrons: A Brand New Pathway to Astronomy, Astrophysics, and Particle Physics, JHEAp 1-2 (2014) 1-30. arXiv:1312.6587. doi:10.1016/j.jheap.2014.01.001

[6] J. G. Learned, S. Pakvasa, Detecting tau-neutrino oscillations at PeV energies, Astropart. Phys. 3 (1995) 267-274. arXiv: hep-ph/9405296 doi:10.1016/0927-6505(94)00043-3

[7] T. Kashti, E. Waxman, Flavoring astrophysical neutrinos: Flavor ratios depend on energy, Phys. Rev. Lett. 95 (2005) 181101. arXiv: astro-ph/0507599 doi:10.1103/PhysRevLett.95.181101

[8] P. Lipari, M. Lusignoli, D. Meloni, Flavor Composition and Energy Spectrum of Astrophysical Neutrinos, Phys. Rev. D75 (2007) 123005. arXiv:0704.0718 doi:10.1103/PhysRevD.75.123005

[9] M. Kachelriess, S. Ostapchenko, R. Tomas, High energy neutrino yields from astrophysical sources. 2. Magnetized sources, Phys. Rev. D77 (2008) 023007. arXiv:0708.3047, doi:10.1103/PhysRevD.77.023007

[10] S. Hummer, M. Maltoni, W. Winter, C. Yaguna, Energy dependent neutrino flavor ratios from cosmic accelerators on the Hillas plot, Astropart. Phys. 34 (2010) 205-224. arXiv:1007.0006 doi:10.1016/j.astropartphys.2010.07.003

[11] W. Winter, Describing the Observed Cosmic Neutrinos by Interactions of Nuclei with Matter, Phys. Rev. D90 (10) (2014) 103003. arXiv:1407.7536 doi:10.1103/PhysRevD.90.103003. 


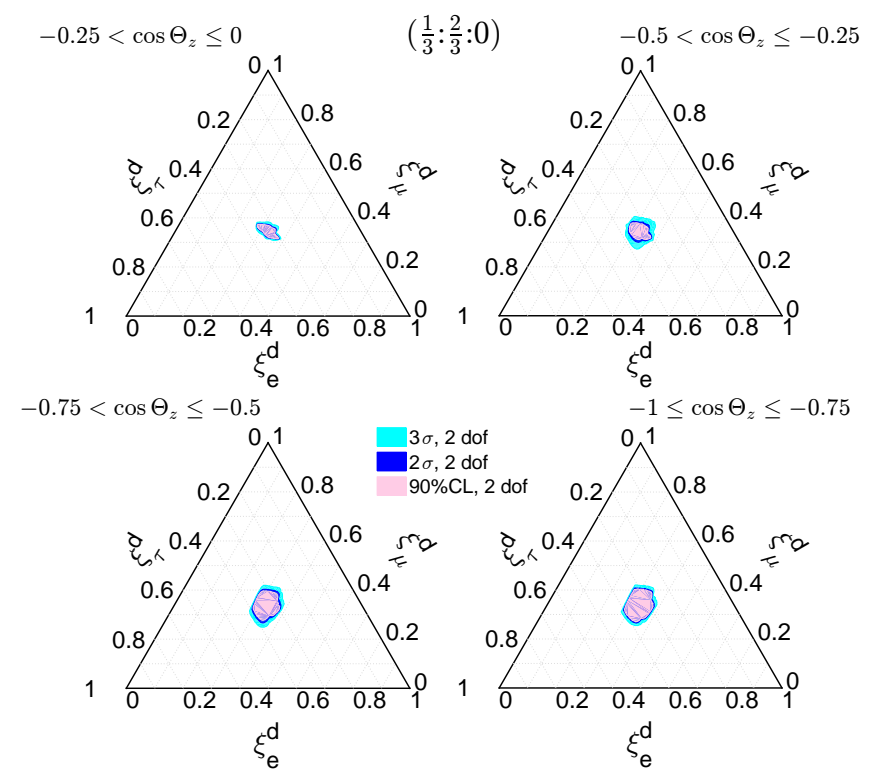

Figure 6: Same as Fig. 4 for $\left(\xi_{e}^{s}: \xi_{\mu}^{s}: \xi_{\tau}^{s}\right)=\left(\frac{1}{3}: \frac{2}{3}: 0\right)$.

[12] C. Lunardini, A. Yu. Smirnov, High-energy neutrino conversion and the lepton asymmetry in the universe, Phys. Rev. D64 (2001) 073006. arXiv:hep-ph/0012056 doi:10.1103/PhysRevD.64.073006

[13] S. Razzaque, A. Yu. Smirnov, Flavor conversion of cosmic neutrinos from hidden jets, JHEP 03 (2010) 031 . arXiv: 0912.4028 doi:10.1007/JHEP03(2010) 031

[14] S. Sahu, B. Zhang, Effect of Resonant Neutrino Oscillation on TeV Neutrino Flavor Ratio from Choked GRBs, Res. Astron. Astrophys. 10 (2010) 943-949. arXiv:1007.4582, doi:10.1088/1674-4527/10/10/001

[15] O. Mena, S. Palomares-Ruiz, A. C. Vincent, Flavor Composition of the High-Energy Neutrino Events in IceCube, Phys. Rev. Lett. 113 (2014) 091103. arXiv:1404.0017, doi:10.1103/PhysRevLett.113.091103

[16] M. Bustamante, J. F. Beacom, W. Winter, Theoretically palatable flavor combinations of astrophysical neutrinos, Phys. Rev. Lett. 115 (16) (2015) 161302. arXiv:1506.02645 doi:10.1103/PhysRevLett.115.161302

[17] A. Palladino, G. Pagliaroli, F. Villante, F. Vissani, What is the Flavor of the Cosmic Neutrinos Seen by IceCube?, Phys. Rev. Lett. 114 (17) (2015) 171101. arXiv:1502.02923 doi:10.1103/PhysRevLett.114.171101

[18] S. Palomares-Ruiz, A. C. Vincent, O. Mena, Spectral analysis of the high-energy IceCube neutrinos, Phys. Rev. D91 (10) (2015) 103008. arXiv:1502.02649, doi:10.1103/PhysRevD.91.103008

[19] A. Watanabe, The spectrum and flavor composition of the astrophysical neutrinos in IceCube, JCAP 1508 (2015) 030. arXiv: 1412.8264 doi:10.1088/1475-7516/2015/08/030

[20] N. Kawanaka, K. Ioka, Neutrino Flavor Ratios Modified by Cosmic Ray Secondary Acceleration, Phys. Rev. D92 (8) (2015) 085047. arXiv:1504.03417, doi:10.1103/PhysRevD.92.085047

[21] M. G. Aartsen, et al., A combined maximum-likelihood analysis of the high-energy astrophysical neutrino flux measured with IceCube, Astrophys. J. 809 (1) (2015) 98. arXiv:1507.03991 doi:10.1088/0004-637X/809/1/98

[22] A. C. Vincent, S. Palomares-Ruiz, O. Mena, Analysis of the 4-year IceCube HESE datdarXiv: 1605.01556.

[23] D. Hooper, D. Morgan, E. Winstanley, Lorentz and CPT invariance violation in high-energy neutrinos, Phys. Rev. D72 (2005) 065009. arXiv:hep-ph/0506091 doi:10.1103/PhysRevD.72.065009

[24] J. F. Beacom, N. F. Bell, D. Hooper, S. Pakvasa, T. J. Weiler, Decay of high-energy astrophysical neutrinos, Phys. Rev. Lett. 90 (2003) 181301. arXiv:hep-ph/0211305, doi:10.1103/PhysRevLett.90.181301

[25] P. Baerwald, M. Bustamante, W. Winter, Neutrino Decays over Cosmological Distances and the Implications for Neutrino Telescopes, JCAP 1210 (2012) 020. arXiv:1208.4600 doi:10.1088/1475-7516/2012/10/020

[26] L. A. Anchordoqui, H. Goldberg, M. C. Gonzalez-Garcia, F. Halzen, D. Hooper, S. Sarkar, T. J. Weiler, Probing Planck scale physics with IceCube, Phys. Rev. D72 (2005) 065019. arXiv:hep-ph/0506168 doi:10.1103/PhysRevD.72.065019

[27] D. Hooper, D. Morgan, E. Winstanley, Probing quantum decoherence with high-energy neutrinos, Phys. Lett. B609 (2005) 206-211. arXiv:hep-ph/0410094 doi:10.1016/j.physletb.2005.01.034

[28] J. F. Beacom, N. F. Bell, D. Hooper, J. G. Learned, S. Pakvasa, T. J. Weiler, PseudoDirac neutrinos: A Challenge for neutrino telescopes, Phys. Rev. Lett. 92 (2004) 011101. arXiv:hep-ph/0307151 doi:10.1103/PhysRevLett.92.011101

[29] A. Esmaili, Pseudo-Dirac Neutrino Scenario: Cosmic Neutrinos at Neutrino Telescopes, Phys. Rev. D81 (2010) 013006. arXiv:0909.5410 doi:10.1103/PhysRevD.81.013006

[30] H. Athar, M. Jezabek, O. Yasuda, Effects of neutrino mixing on high-energy cosmic neutrino flux, Phys. Rev. D62 (2000) 103007. arXiv:hep-ph/0005104 doi:10.1103/PhysRevD.62.103007

[31] P. F. de Salas, R. A. Lineros, M. Trtola, Neutrino propagation in the galactic dark matter haloarXiv: 1601.05798 
[32] C. A. Argelles, T. Katori, J. Salvado, New Physics in Astrophysical Neutrino Flavor, Phys. Rev. Lett. 115 (2015) 161303. arXiv:1506.02043, doi:10.1103/PhysRevLett.115.161303

[33] Z. Maki, M. Nakagawa, S. Sakata, Remarks on the unified model of elementary particles, Prog. Theor. Phys. 28 (1962) 870-880. doi:10.1143/PTP.28.870

[34] M. Kobayashi, T. Maskawa, CP Violation in the Renormalizable Theory of Weak Interaction, Prog. Theor. Phys. 49 (1973) 652-657. doi:10.1143/PTP.49.652

[35] M. C. Gonzalez-Garcia, M. Maltoni, Determination of matter potential from global analysis of neutrino oscillation data, JHEP 09 (2013) 152. arXiv:1307.3092 doi:10.1007/JHEP09(2013) 152

[36] A. Dziewonski, D. Anderson, Preliminary reference earth model, Phys.Earth Planet.Interiors 25 (1981) 297-356. doi: 10.1016/0031-9201(81) 90046-7.

[37] I. M. Shoemaker, K. Murase, Probing BSM Neutrino Physics with Flavor and Spectral Distortions: Prospects for Future High-Energy Neutrino Telescopes, Phys. Rev. D93 (8) (2016) 085004. arXiv:1512.07228 doi:10.1103/PhysRevD.93. 085004 .

[38] H. Nunokawa, B. Panes, R. Z. Funchal, How Unequal Fluxes of High Energy Astrophysical Neutrinos and Antineutrinos can Fake New Physic: arXiv: 1604.08595

[39] J. Jones, I. Mocioiu, M. H. Reno, I. Sarcevic, Tracing very high-energy neutrinos from cosmological distances in ice, Phys. Rev. D69 (2004) 033004. arXiv:hep-ph/0308042 doi:10.1103/PhysRevD.69.033004

[40] M. C. Gonzalez-Garcia, F. Halzen, M. Maltoni, Physics reach of high-energy and high-statistics icecube atmospheric neutrino data, Phys. Rev. D71 (2005) 093010. arXiv:hep-ph/0502223 doi:10.1103/PhysRevD.71.093010

[41] C. A. Argelles Delgado, J. Salvado, C. N. Weaver, A Simple Quantum Integro-Differential Solver (SQuIDS), Comput. Phys. Commun. 196 (2015) 569-591. arXiv:1412.3832 doi:10.1016/j.cpc.2015.06.022

[42] M. C. Gonzalez-Garcia, M. Maltoni, J. Salvado, Testing matter effects in propagation of atmospheric and long-baseline neutrinos, JHEP 05 (2011) 075. arXiv:1103.4365, doi:10.1007/JHEP05(2011) 075

[43] O. Miranda, M. Tortola, J. Valle, Are solar neutrino oscillations robust?, JHEP 0610 (2006) 008. arXiv:hep-ph/0406280, doi: 10.1088/1126-6708/2006/10/008

[44] S. Davidson, C. Pena-Garay, N. Rius, A. Santamaria, Present and future bounds on nonstandard neutrino interactions, JHEP 0303 (2003) 011. arXiv: hep-ph/0302093.

[45] C. Biggio, M. Blennow, E. Fernandez-Martinez, General bounds on non-standard neutrino interactions, JHEP 0908 (2009) 090. arXiv:0907.0097, doi:10.1088/1126-6708/2009/08/090

[46] J. Dorenbosch, et al., Experimental verification of the universality of electron-neutrino and muon-neutrino coupling to the neutral weak current, Phys.Lett. B180 (1986) 303. doi:10.1016/0370-2693(86)90315-1

[47] J. Allaby, et al., A Precise Determination of the Electroweak Mixing Angle from Semileptonic Neutrino Scattering, Z.Phys. C36 (1987) 611. doi:10.1007/BF01630598

[48] A. Blondel, P. Bockmann, H. Burkhardt, F. Dydak, A. Grant, et al., Electroweak parameters from a high statistics neutrino nucleon scattering experiment, Z.Phys. C45 (1990) 361-379. doi:10.1007/BF01549665

[49] G. Zeller, et al., A Precise determination of electroweak parameters in neutrino nucleon scattering, Phys.Rev.Lett. 88 (2002) 091802. arXiv:hep-ex/0110059 doi:10.1103/PhysRevLett.88.091802

[50] M. C. Gonzalez-Garcia, M. Maltoni, T. Schwetz, Updated fit to three neutrino mixing: status of leptonic CP violation, JHEP 11 (2014) 052. arXiv:1409.5439 doi:10.1007/JHEP11(2014)052

[51] M. Gonzalez-Garcia, M. Maltoni, T. Schwetz, NuFit 2.0 (2014), http://www.nu-fit.org

[52] A. Palladino, F. Vissani, The natural parameterization of cosmic neutrino oscillations, Eur. Phys. J. C75 (2015) 433. arXiv:1504.05238, doi:10.1140/epjc/s10052-015-3664-6 